\title{
La transmisión de valores sociales a través de Internet
}

\author{
Paula Renés-Arellano ${ }^{1}$, Cleofé Genoveva Alvites-Huamaní y Mari-Carmen Caldeiro-Pedreira ${ }^{3}$ \\ ${ }^{1}$ Universidad de Cantabria (España), ${ }^{2}$ Universidad Alas Peruanas (Perú), ${ }^{3}$ Universidad de Santiago de Compostela (España)
}

\section{RESUMEN}

La presencia de Internet y la necesidad de comprender cómo afecta su uso en la adquisición de valores sociales en el contexto universitario, han promovido la realización de esta investigación. El objetivo general es analizar las relaciones entre la presencia de valores sociales y la utilización de Internet de los estudiantes universitarios. El estudio de corte descriptivo presenta un diseño no experimental, con una muestra de 305 estudiantes de tres universidades españolas y una chilena, adscritas a las facultades de educación y ciencias humanas y sociales. Los resultados han contrastado la hipótesis general relacionando la presencia de valores sociales como resultado de fines comunicativos en los estudiantes universitarios, $(p=0.011)$. Asimismo, se han contrastado las hipótesis específicas. En la primera (H1), la presencia de valores sociales se relaciona significativamente con la dimensión relevancia del uso de Internet $(p=0.037<0.05)$. En la segunda (H2), la presencia de valores sociales se relaciona significativamente con los años de uso de Internet en los estudiantes $(p=0.001<0.05)$. En la tercera $(\mathrm{H} 3)$, el uso de Internet en horas a la semana se relaciona con que Internet transmite valores $(p=0.048<0.05)$. Y en la cuarta (H4), la dimensión finalidad de uso de Internet para obtener información, comunicarse y entretenimiento de los estudiantes se relaciona con que Internet transmite valores sociales $(p=0.03<0.05)$.

Palabras Clave: Educación Superior, Internet, comunicación, valores sociales, percepción del alumnado.

\section{The transmission of social values through the Internet abstract}

\begin{abstract}
The presence of the Internet and the need to understand how its use affects the acquisition of social values in the university context, have promoted the conduct of this research. The general objective is to analyze the relationships between the presence of social values and the use of the Internet by university students. The descriptive study presents a non-experimental design, with a sample of 305 students from three Spanish universities and one Chilean, Chilean, attached to the faculties of education and human and social sciences. The results have contrasted the general hypothesis related to the fact that the presence of social values is given for communicative purposes in university students, $(p=0.011)$. Also, the specific hypotheses have been contrasted. In the first (H1), the presence of social values is It is significantly related to the relevance dimension of Internet use $(p=0.037<0.05)$. In the second (H2), the presence of social values is significantly related to the years of Internet use in students $(p=0.001<0.05)$. In the third (H3), the use of the Internet in hours per week is related to the fact that the Internet transmits values $(p=0.048<0.05)$. And in the fourth $(\mathrm{H} 4)$, the purpose dimension of Internet use to obtain information, communicate and entertain students is related to the fact that the Internet transmits social values $(p=0.03<0.05)$.
\end{abstract}

Keywords: Higher Education, Internet, communication, social values, perception of students. 


\section{Introducción}

En la sociedad digital resulta incuestionable la presencia de Internet, una presencia que se agudiza, de forma notable entre la población juvenil y adulta, pero, sobre todo, entre los estudiantes universitarios. Tanto los millennials, como la Generación Z (Atrevia, 2016), también denominada Generación Google (Parodi, Moreno de León, Julio y Burdiles, 2019) han nacido al lado de la tecnología, un hecho que favorece su uso, en ocasiones indiscriminado y hasta cierto punto inconsciente. La población más joven de forma habitual emplea los recursos digitales, las redes sociales o las plataformas virtuales para comunicarse, relacionarse, formarse o informarse, pero, sobre todo, para interpretar el mundo que les rodea (Lévi, 1999; Smith \& Kollock, 2003; Bauman, 2014; Fombona, Goulao y García, 2014). De forma general, puede afirmarse que buscan hacer de éste, un lugar en el que entender quiénes son y qué función tienen como personas y ciudadanos.

El ecosistema actual permite a los estudiantes convivir en espacios naturales, urbanos y virtuales (Echeverría, 2000), por lo que las formas de comunicarse varían. Esta nueva cultura mediática, digital o tecnologizada, propia de la Sociedad Red, está generando entre los jóvenes nuevos modelos comunicativos, de interacción y de comprensión de las realidades (Colom y Melich, 1993, Garrido-Cabezas, 2011). En este contexto, resulta compleja la transmisión de unos valores que respondan adecuadamente a las demandas, necesidades e interpretaciones personales y sociales, en especial entre los colectivos más jóvenes.

Concretamente, en el ámbito universitario, estos estudiantes denominados, como hemos indicado anteriormente, millennians o generación on-off, demandan nuevas destrezas, competencias y objetivos curriculares que requieren metodologías activas, participativas y enmarcados en los ecosistemas digitales en los que viven. En esta línea, las universidades españolas, europeas o iberoamericanas ya incorporan nuevos modelos educativos adaptados a este contexto, como describen Silva y Maturana (2017) en relación con el empleo de nuevas metodologías activas en educación superior. Formas de enseñar adecuadas a las necesidades del S.XXI, tales como las que pretende el planteamiento docente del programa educativo de la Fundación Universidad de La Rioja que busca, según refleja en la Web, "Incorporar, de forma gradual, el uso de las nuevas tecnologías de la información y la comunicación en el desarrollo de la docencia". En esta línea, en Colombia se ha hecho uso del vídeo educativo en Educación Superior (Ricardo e Iriarte, 2017). Asimismo, también destaca el Informe publicado por Adams-Becker, et al., (2017) sobre las tendencias tecnológicas en educación superior empleando metodologías mixtas y colaborativas en las aulas, y destacando el proyecto piloto realizado por la Universidad de Sydney para que estudiantes universitarios trabajen de forma conjunta en ideas y financiaciones para las mismas. Posteriormente, se destaca el informe Horizon 2019 (Alexander, et al, 2019), en el que se apuesta por el desarrollo e implementación de formaciones e-learning, un aspecto cultural hacia el que se dirigen las tendencias en educación superior para 2023. Sin embargo, en este nuevo panorama no solo es importante atender las metodologías innovadoras sino que también es fundamental realizar investigaciones con los jóvenes (Palfrey \& Gasser, 2008), que miren desde dentro, que ayuden a conocer las verdaderas percepciones de los estudiantes universitarios sobre cómo conciben ellos el entorno de Internet, así como los valores sociales que se producen y nacen de estos usos, porque los contenidos mediáticos promueven nuevos hábitos comunicativos cargados de valores e ideologías (Figueras, Ferrés y Mateus, 2018). Autores como Carmen y Agarwal (2002) o Reig (2018) señalan aspectos positivos sobre el uso de Internet y la construcción de valores, defendiendo que el control y comprensión de los diferentes canales comunicativos en las redes, exige el desarrollo de determinadas habilidades y destrezas. A ello, se suma la relevancia, no solo de dichas competencias técnicas, operativas o procedimentales, sino también actitudinales, porque en ese mundo de interrelaciones virtuales emergen los valores propios que permiten relacionarnos y actuar en consonancia a estos. Porque todas las relaciones humanas están "mediadas por valores, comportamientos y actitudes sobre los que no se opera de la misma manera como con los conceptos o los procedimientos, sino como reguladores de las operaciones" (Álvarez-Arregui, 2019). Es, en este contexto, donde resulta complejo conocer realmente qué valores sociales son los que se promueven en Internet (Orantes, 2011). En esta línea, autores como Tornero (2017) o Anaya (2019) se cuestionan qué tipo de modelos éticos existen en el mundo digital, en el que conviven millones de personas, pero en el que también, algunas de ellas, encuentran su espacio para desarrollar sus competencias sociales cuando no son capaces de hacerlo en el contacto cotidiano, los denominados "solitarios electrónicos" (Gubern, 2000). Desde esta perspectiva, parece necesario abordar cómo y de qué manera desde el campo educativo universitario se puede promover el desarrollo de ciertas competencias socioemocionales y comunicativas que permitan a los estudiantes desenvolver sus propias capacidades, destrezas y valores individuales y sociales. Asimismo, resulta clave que la población y en especial el colectivo joven, sea capaz de analizar de forma crítica y reflexiva la ingente cantidad de información que recibe (Caldeiro-Pedreira, 2014). Para ello, es fundamental el desarrollo de la competencia crítica (Caldeiro y Aguaded-Gómez, 2015) que favorece el análisis y la reflexión por parte del usuario que debe abandonar el papel pasivo para convertirse en un receptor activo y crítico con los valores que reflejan los contenidos digitales que se emiten a través de los diferentes dispositivos y medios tecnológicos. En este sentido, es necesario que la población usuaria de Internet y de los diferentes medios actúe como prosumidora y sea capaz de interactuar (Berlanga, Gozálvez, Renés-Arellano y Aguaded, 2018).

Indudablemente, en el marco descrito, Internet se ha convertido en un espacio de construcción de valores sociales compartidos y eso exige una reflexión responsable (Aparici, 2010). Si se desea favorecer una adecuada formación entre el alumnado universitario es relevante plantearse un nuevo reto de adaptación a las nuevas exigencias contextuales, personales y sociales (Boyer, 2003). Lo cierto es que a pesar de los riesgos que puede suponer el uso de Internet entre los jóvenes, tal y como afirman Asher, Stark \& Fireman (2017) sobre el acoso electrónico entre poblaciones universitarias, o Guerra et al., (2019), sobre un estudio preliminar acerca del riesgo indirecto de victimización en el espacio virtual, también existen investigaciones que demuestran los beneficios de la utilización de Internet entre jóvenes en el plano académico, tales como las de Acuña Caicedo, Caicedo-Plúa, Rodríguez y Figueroa Morán (2017), quienes afirman que favorece la formación y relaciones a través de herramientas como pueden ser los MOOC. Por su parte, DiMaggio et al., (2004) comparten la idea de que el uso de Internet permite mejorar los espacios recreativos y con ello, el desarrollo de competencias personales y sociales y la mejora de la percepción personal; y, Hassani (2006) defiende que, a mayor grado de utilización de Internet en diversas actividades, se observaba una mejor autonomía, y esto a su vez, exige el desarrollo y potencialización de habilidades sociales (Wilson, 2000).

De forma general puede aseverarse que, Internet está presente y además influye positiva o negativamente en las relaciones entre los estudiantes universitarios y en la adquisición de sus propios 
valores, por lo que resulta fundamental promover modelos comunicativos y educativos que hagan que los jóvenes sean conscientes y responsables del uso del mismo, porque si bien Internet puede permitir el acercamiento a interacciones sociales nuevas o ya existentes, de una $\mathrm{u}$ otra manera, nos expone al resto de personas (Ibarra, Ballester y Marín, 2018). Es por ello, que desde el contexto universitario se requiere reflexionar críticamente sobre cómo los estudiantes universitarios están desarrollando y forjando su propio juicio moral, sus valores personales y sociales sustentados en principios éticos, democráticos y participativos (Gozálvez, 2013). En este sentido, resulta clave señalar que, los valores de una sociedad se convierten en pautas de acción individual que son aceptadas por todos y que contribuyen activamente al sentimiento de pertenencia a un grupo, de aceptación y respeto a los demás (Hernández y Eyeang, 2017). Además de ello, los valores sociales son asumidos por cada persona, no solo por ser característicos de un grupo social o contexto, sino porque realmente se aceptan como válidos y relevantes en el momento en el que son observados y estimados como tal (Martínez, Esteban y Buxarrais, 2011).

En el contexto actual, la complejidad derivada de esta nueva cultura exige modelos educativos en los que se propicie la construcción de valores individuales y sociales que contribuyan al desarrollo de una ciudadanía con principios dialógicos, participativos, democráticos y responsables (UNESCO, 2015). Ante esta situación, el profesorado universitario puede plantearse interrogantes sobre cómo el alumnado universitario percibe Internet en su día a día, con el fin de adecuarse a los patrones personales y sociales en los que conviven, para responder a las demandas académicas, individuales y sociales, y para promover procesos de enseñanza y aprendizaje adaptados a los nuevos modelos comunicativos, académicos y laborales.

Por todo ello, es el momento de conocer cómo perciben los valores los estudiantes universitarios, cómo los consumen y son asimilados a través de Internet (Colina, 2012). En esta línea, Martínez-Otero Pérez (2019), estudia la relación entre valores e ideologías políticas, categorizando la presencia o ausencia de valores de la siguiente manera: cognitivos e intelectuales, artísticos/estéticos, morales/éticos, trascendentales, espirituales y religiosos; afectivo emocionales, socioculturales, físicos, económicos y ecológicos, destacando los valores sociales y éticos como aquellos que fueron más compartidos entre la muestra seleccionada de diferentes países, y destacando la amplia pluralidad axiológica vinculada a la educación y la política.

Contextualizada la importancia de los valores en la Sociedad Red, se plantea el siguiente estudio, con la finalidad de profundizar en las diversas relaciones existentes entre el empleo de Internet por parte del alumnado universitario y la presencia de valores, concretamente, sociales.

\section{Materiales y métodos}

\subsection{Tipo y Diseño}

El tipo de estudio es descriptivo, con diseño no experimental debido a que no se manipulan las variables observando como ocurre de manera natural sin intervenir de manera alguna por lo que solo es posible investigar asociaciones, además es transeccional ya que se realiza en un momento determinado o tiempo único y correlacional porque se busca analizar relaciones entre variables (Beaumont, 2009).

Teniendo en cuenta lo señalado hasta el momento, el estudio plantea un objetivo general y objetivos específicos. Siendo el general: Analizar las múltiples relaciones entre la presencia de valores sociales y la utilización de Internet en estudiantes universitarios.

Por su parte, se proponen los siguientes objetivos específicos: i. Determinar la relación entre la presencia de valores sociales y la relevancia de uso de Internet en estudiantes universitarios; ii. Determinar la relación entre la presencia de valores sociales y los años de uso de Internet en estudiantes universitarios; iii. Determinar la relación entre la presencia de valores sociales y las horas a la semana de uso de Internet en estudiantes universitarios; y, iv. Determinar la relación entre la presencia de valores sociales y la finalidad de uso de Internet en estudiantes universitarios.

Por lo que, en este estudio se planteó como hipótesis general: Los estudiantes que utilizan Internet presentan más valores sociales. Y como Hipótesis específicas: (H1) La presencia de valores sociales se relaciona con la relevancia del uso de Internet en los estudiantes; (H2) Los años de uso de Internet cimenta los valores sociales en los estudiantes; (H3) La presencia de valores en Internet se relaciona con las horas a la semana de uso de Internet en los estudiantes; y (H4) Los estudiantes que usan Internet con la finalidad de obtener información, comunicarse y entretenimiento presentan más valores sociales.

\subsection{Participantes}

La muestra de estudio estuvo compuesta por 305 estudiantes provenientes de tres universidades públicas españolas y una universidad chilena, todas adscritas a las facultades de educación y ciencias humanas y sociales (véase tabla 1). Los estudiantes participantes estaban cursando el primer y segundo ciclo de estudios, siendo la edad con mayor porcentaje menores a 20 años (59\%) seguida de 20 a 30 años (39\%) y un pequeño porcentaje mayores de 35 años $(2 \%)$, en su mayoría de género femenino $(85 \%)$.

Tabla 1

Muestra de estudio en porcentajes

\begin{tabular}{lcccccc}
\hline Genero & $\begin{array}{c}\% \text { de } \\
\text { estudiantes }\end{array}$ & Edad & $\begin{array}{c}\% \text { de } \\
\text { estudiantes }\end{array}$ & $\begin{array}{c}\text { Facultad } \\
\text { de } \\
\text { procedencia }\end{array}$ & $\begin{array}{c}\text { \% de } \\
\text { estudiantes }\end{array}$ & $\begin{array}{c}\text { Curso de } \\
\text { académico } \\
\text { estudiantes }\end{array}$ \\
\hline Masculino & $15 \%$ & $\begin{array}{c}\text { Menores } \\
20 \text { años }\end{array}$ & $59 \%$ & Educación & $3 \%$ & Primero \\
Femenino & $85 \%$ & $\begin{array}{c}20 \text { a } 30 \\
\text { años }\end{array}$ & $39 \%$ & $\begin{array}{c}\text { Ciencias humanas } \\
\text { y sociales }\end{array}$ & $97 \%$ & Segundo \\
& Mayores 35 años & $2 \%$ & & & \\
\hline
\end{tabular}

Fuente: Elaboración propia (2019) 


\subsection{Instrumento}

Para la recolección de la información se aplicaron dos instrumentos vía web.

Para la variable presencia de valores sociales (véase Tabla 2) se elaboró el cuestionario de Valores sociales, conformado por ítems, en escala de Likert, y dividido en dimensiones.

Tabla 2

Descripción del instrumento de la variable presencia de valores sociales

\begin{tabular}{ll}
\hline \multicolumn{1}{c}{ DIMENSIÓN } & \multicolumn{1}{c}{ ITEMS } \\
\hline $\begin{array}{l}\text { Internet transmite valores } \\
\text { Internet promueve valores }\end{array}$ & $2,3,4,5,6,7,8,9,10$, \\
$\begin{array}{l}\text { Identificación de valores con com- } \\
\text { pañeros en la comunicación por }\end{array}$ & $11,12,13,14,15,16,17,18,19$, \\
$\begin{array}{l}\text { Internet } \\
\begin{array}{l}\text { Identificación de valores con amigos } \\
\text { en la comunicación por Internet }\end{array}\end{array}$ & $20,21,22,23,24,25,26,27,28$, \\
$\begin{array}{l}\text { Identificación de valores con familia- } \\
\text { res en la comunicación por Internet }\end{array}$ & $29,30,31,32,33,34,35,36,37$, \\
\hline
\end{tabular}

Fuente: Elaboración propia (2019)

La confiabilidad del cuestionario se realizó mediante el análisis estadístico Alfa de Cronbach, obteniendo en cada una de las dimensiones una fiabilidad de valor entre 0.8 y 0.9 , lo que indica que el instrumento es aceptable a muy aceptable, siendo la dimensión Identificación de valores con familiares en la comunicación por Internet la que obtuvo un puntaje más alto de fiabilidad $(0,9)$ (Véase tabla 3).

Tabla 3

Análisis de fiabilidad de las dimensiones de la variable presencia de valores sociales

\begin{tabular}{ccccc}
\hline Dimensión & $\begin{array}{c}\text { Alfa de } \\
\text { Cronbach }\end{array}$ & $\begin{array}{c}\text { N de } \\
\text { ítems }\end{array}$ & Media & $\begin{array}{c}\text { Desviación } \\
\text { Estándar }\end{array}$ \\
\hline $\begin{array}{c}\text { Internet transmite y } \\
\text { promueve valores } \\
\begin{array}{c}\text { Identificación de } \\
\text { valores con compa- }\end{array}\end{array}$ & 0.802 & 10 & 22.87 & 5.128 \\
$\begin{array}{c}\text { neros en la comuni- } \\
\text { cación por Internet }\end{array}$ & 0.873 & 9 & 29.82 & 5.117 \\
$\begin{array}{c}\text { Identificación de } \\
\text { valores con amigos } \\
\text { en la comunicación } \\
\text { por Internet }\end{array}$ & 0.896 & 9 & 31.35 & 5.169 \\
$\begin{array}{c}\text { Identificación de va- } \\
\text { lores con familiares } \\
\text { en la comunicación } \\
\text { por Internet }\end{array}$ & 0.900 & 9 & 30.76 & 5.918 \\
\hline
\end{tabular}

Fuente: Elaboración propia (2019)

Para la variable utilización de Internet (véase tabla 4) se elaboró el cuestionario "Uso de Internet", con un total de 6 ítems, de escala ordinal, al ser estas dimensiones cualitativas no se requirió contrastar la fiabilidad de la prueba.
Tabla 4

Descripción del instrumento de la variable utilización de Internet

\begin{tabular}{lc}
\hline \multicolumn{1}{c}{ DIMENSIÓN } & ITEMS \\
\hline Relevancia de uso de Internet & 38 \\
Años de uso de Internet & 39 \\
Horas a la semana de uso de Internet & 40 \\
Finalidad de uso de Internet para obtener información & 41 \\
Finalidad de uso de Internet para comunicarse & 42 \\
Finalidad de uso de Internet para entretenimiento & 43 \\
\hline
\end{tabular}

Fuente: Elaboración propia (2019)

\subsection{Procedimiento}

Antes de la obtención de los resultados, se informó a las instituciones universitarias de la realización de este estudio, contando así mismo, con la aprobación de los estudiantes universitarios, quienes de forma voluntaria, informada y confidencial participaron en el desarrollo de la investigación. Una vez obtenidos los resultados de los cuestionarios completados por los estudiantes en Excel, fueron ordenados y categorizados por una baremación para el test de la presencia de valores en Internet.

Posteriormente, se analizaron las relaciones entre variables por medio de la prueba chi-cuadrado, t-Student y ANOVA en SPSS v20. Se rechazó la hipótesis nula para cada hipótesis planteada cuando se observó un valor $p<0.05$.

Las dimensiones relevancia de uso de Internet, finalidad de uso de Internet para obtener información, finalidad de uso de Internet para comunicar y finalidad de uso de Internet para entretenimiento fueron estudiadas como variables categóricas; de igual manera que con todas las dimensiones de la variable presencia de valores por lo que se analizó con la prueba chi-cuadrado. Mientras que las dimensiones años de uso de Internet y horas a la semana fueron considerada como variables cuantitativas por lo que se realizó el análisis de t de student (estadístico t) para comparar a los 2 grupos y ANOVA (estadístico F) cuando se comparan entre más de 2 grupos.

Para la prueba de fiabilidad para el cuestionario de presencia de valores sociales, se realizó la prueba alfa de Cronbach para cada una de las dimensiones.

\section{Resultados}

En relación con los resultados del estudio, se inició la descripción y análisis de estos a partir de las hipótesis planteadas y el tratamiento de los datos obtenidos, destacando en primer lugar, la evaluación realizada en la tabla 5, en la que se observó que la identificación de valores en general se relaciona significativamente con la dimensión finalidad de uso de Internet para comunicarse $(p=0.011)$. Es por ello, que se desestima la hipótesis nula $\left(\mathrm{H}_{\mathrm{o}}\right)$ y se acepta la hipótesis general: la presencia de valores sociales se relaciona con la utilización de Internet, y en concreto, con fines comunicativos. 
Tabla 5

Análisis presencia de valores sociales y la utilización de Internet

\begin{tabular}{|c|c|c|c|c|c|c|c|c|c|c|c|c|}
\hline \multirow{3}{*}{$\begin{array}{l}\text { Presencia } \\
\text { de valores } \\
\text { en Internet }\end{array}$} & \multicolumn{12}{|c|}{ Uso de Internet } \\
\hline & \multicolumn{2}{|c|}{$\begin{array}{l}\text { Relevancia de uso de } \\
\text { Internet }\end{array}$} & \multicolumn{2}{|c|}{$\begin{array}{l}\text { Años de uso de } \\
\text { Internet }\end{array}$} & \multicolumn{2}{|c|}{$\begin{array}{c}\text { Horas a la } \\
\text { semana de } \\
\text { Internet }\end{array}$} & \multicolumn{2}{|c|}{$\begin{array}{l}\text { Finalidad de uso de } \\
\text { Internet para obtener } \\
\text { información }\end{array}$} & \multicolumn{2}{|c|}{$\begin{array}{c}\text { Finalidad de uso de } \\
\text { Internet para comu- } \\
\text { nicarse } \\
\end{array}$} & \multicolumn{2}{|c|}{$\begin{array}{c}\text { Finalidad de uso de } \\
\text { Internet para } \\
\text { entretenimiento }\end{array}$} \\
\hline & Chi-cuadrado & Sig. & $\mathrm{F}$ & Sig. & $\mathrm{F}$ & Sig. & Chi-cuadrado & Sig. & Chi-cuadrado & Sig. & Chi-cuadrado & Sig. \\
\hline $\begin{array}{l}\text { Identificación de } \\
\text { valores (general) }\end{array}$ & 1.942 & 0.379 & 0.123 & 0.885 & 0.610 & 0.544 & 1.900 & 0.754 & 13.135 & $0.011^{*}$ & 5.539 & 0.477 \\
\hline
\end{tabular}

* $\mathrm{p}<0.05$ diferencia significativa

Fuente: Elaboración propia (2019)

Atendiendo a la evaluación de la siguiente tabla en la que se analiza la presencia de valores sociales y la dimensión utilización de Internet (véase tabla 6), se observó que la dimensión "relevancia de uso de Internet" se relaciona significativamente con la afirmación de que el Internet transmite valores $(p=0.037<0.05)$. No habiendo encontrado relaciones entre las demás dimensiones de presencia de valores sociales, se desestima la hipótesis nula y se acepta la hipótesis específica, es decir, que la presencia de valores sociales se relaciona significativamente con la dimensión relevancia del uso de Internet en los estudiantes (H1).

Tabla 6

Análisis de la presencia de valores sociales y la dimensión de relevancia de uso de Internet

\begin{tabular}{|c|c|c|}
\hline \multirow{2}{*}{$\begin{array}{l}\text { Presencia de valores sociales } \\
\text { Dimensiones }\end{array}$} & \multicolumn{2}{|c|}{$\begin{array}{c}\text { Relevancia } \\
\text { de uso de Internet }\end{array}$} \\
\hline & Chi-cuadrado & Sig. \\
\hline Internet transmite valores & 4.329 & $0.037^{*}$ \\
\hline Internet promueve valores & 2.864 & 0.239 \\
\hline $\begin{array}{l}\text { Identificación de valores con com- } \\
\text { pañeros en la comunicación por } \\
\text { Internet }\end{array}$ & 0.952 & 0.621 \\
\hline $\begin{array}{l}\text { Identificación de valores con } \\
\text { amigos en la comunicación por } \\
\text { Internet }\end{array}$ & 2.654 & 0.265 \\
\hline $\begin{array}{l}\text { Identificación de valores con fa- } \\
\text { miliares en la comunicación por } \\
\text { Internet }\end{array}$ & 0.923 & 0.630 \\
\hline
\end{tabular}

* $\mathrm{p}<0.05$ diferencia significativa

Fuente: Elaboración propia (2019)

En relación con la evaluación realizada en la tabla 7, en la cual se analizó la presencia de valores sociales y la dimensión años de uso de Internet, se observa que existe relación altamente significativa en la dimensión "Internet transmite valores" $(p=0.001<0.05)$ y la utilización de Internet en la dimensión años de uso de Internet. No habiendo encontrado relaciones entre las demás dimensiones de presencia de valores sociales. Por lo que, se desestima la hipótesis nula $\left(\mathrm{H}_{\mathrm{o}}\right)$ y se acepta la hipótesis específica de que la presencia de valores sociales se relaciona significativamente con los años de uso de Internet en los estudiantes (H2).
Tabla 7

Análisis presencia de valores sociales y la dimensión años de uso de Internet

\begin{tabular}{lcc}
\hline \multicolumn{1}{c}{ Presencia de valores sociales } & \multicolumn{2}{c}{ Años de uso de Internet } \\
\hline Dimensiones & t y F & Sig. \\
Internet transmite valores & -3.597 & $0.001^{*}$ \\
Internet promueve valores & 1.875 & 0.155 \\
$\begin{array}{l}\text { Identificación de valores con com- } \\
\text { pañeros en la comunicación por }\end{array}$ & 1.758 & 0.174 \\
$\begin{array}{l}\text { Internet } \\
\text { Identificación de valores con ami- } \\
\text { gos en la comunicación por Internet }\end{array}$ & 2.447 & \\
$\begin{array}{l}\text { Identificación de valores con fa- } \\
\text { miliares en la comunicación por }\end{array}$ & & 0.088 \\
$\begin{array}{l}\text { Internet } \\
\text { * p <0.05 diferencia significativa }\end{array}$ & & \\
Fuente: Elaboración propia (2019) & & \\
\end{tabular}

Así mismo, observando la evaluación realizada en la tabla 8, en la cual se analizó la presencia de valores sociales y horas de uso de Internet, se observó que existe relación con que Internet transmite valores $(p=0.048<0.05)$ y la utilización de Internet en la dimensión horas a la semana de uso de Internet. No habiendo encontrado relaciones entre las demás dimensiones de presencia de valores sociales. Por lo que, se desestima la hipótesis nula $\left(\mathrm{H}_{\mathrm{o}}\right)$ y se acepta la hipótesis específica de que la presencia de valores en Internet se relaciona significativamente con las horas a la semana de uso de Internet en los estudiantes (H3).

Tabla 8

Análisis de valores sociales y las horas a la semana de uso de Internet

\begin{tabular}{llc}
\hline \multicolumn{1}{c}{ Presencia de valores sociales } & \multicolumn{2}{c}{$\begin{array}{c}\text { Horas a la semana } \\
\text { de uso de Internet }\end{array}$} \\
\hline Dimensiones & t y F & Sig. \\
Internet transmite valores & -2.006 & $0.048^{*}$ \\
Internet promueve valores & 2.157 & 0.117
\end{tabular}


Identificación de valores con

compañeros en la comunicación

0.291

0.748

por Internet

Identificación de valores con

amigos en la comunicación por

1.772

Internet

\section{Identificación de valores con}

familiares en la comunicación

2.015

0.135

por Internet

* $\mathrm{p}<0.05$ diferencia significativa

Fuente: Elaboración propia (2019)

Tomando en consideración la evaluación realizada en la tabla 9, en la cual se analizó la presencia de valores sociales y las dimensiones finalidad de uso de Internet para obtener información, para comunicarse y para entretenimiento de los estudiantes, se observó que la utilización de Internet de la dimensión finalidad de uso de Internet para obtener información se relaciona con que Internet transmite valores sociales $(p=0.03)$. Por lo que, se desestima la hipótesis nula $\left(\mathrm{H}_{\mathrm{o}}\right)$ y se acepta la hipótesis específica de que la presencia de valores sociales se relaciona significativamente con la finalidad de uso de Internet para obtener información, comunicarse y entretenimiento de los estudiantes (H4).

\section{Tabla 9}

Análisis presencia de valores sociales y la dimensión finalidad de uso de Internet para obtener información, comunicarse y entretenimiento de los estudiantes

\begin{tabular}{|c|c|c|c|c|c|c|}
\hline \multirow{2}{*}{$\begin{array}{c}\text { Presencia } \\
\text { de valores } \\
\text { sociales }\end{array}$} & \multicolumn{6}{|c|}{ Finalidad de uso de Internet } \\
\hline & $\begin{array}{l}\text { Para ob } \\
\text { inform }\end{array}$ & $\begin{array}{l}\text { tener } \\
\text { lación }\end{array}$ & $\begin{array}{r}P a \\
\text { comun }\end{array}$ & $\begin{array}{l}\text { ra } \\
\text { icarse }\end{array}$ & $\begin{array}{r}\mathrm{Pa} \\
\text { entrete }\end{array}$ & $\begin{array}{l}\text { a } \\
\text { nerse }\end{array}$ \\
\hline Dimensiones & $\begin{array}{c}\text { Chi-cua- } \\
\text { drado }\end{array}$ & Sig. & $\begin{array}{c}\text { Chi-cua- } \\
\text { drado }\end{array}$ & Sig. & $\begin{array}{c}\text { Chi-cua- } \\
\text { drado }\end{array}$ & Sig. \\
\hline $\begin{array}{l}\text { Internet transmite } \\
\text { valores }\end{array}$ & 7.022 & $0.03^{*}$ & 0.858 & 0.651 & 6.836 & 0.077 \\
\hline $\begin{array}{l}\text { Internet promueve } \\
\text { valores }\end{array}$ & 7.497 & 0.112 & 12.786 & $0.012^{*}$ & 3.679 & 0.720 \\
\hline $\begin{array}{l}\text { Identificación } \\
\text { de valores con } \\
\text { compañeros en la } \\
\text { comunicación por } \\
\text { Internet }\end{array}$ & 2.510 & 0.643 & 21.780 & $0.000^{*}$ & 10.609 & 0.101 \\
\hline $\begin{array}{l}\text { Identificación de } \\
\text { valores con amigos } \\
\text { en la comunicación } \\
\text { por Internet }\end{array}$ & 10.507 & $0.033^{*}$ & 10.905 & $0.028^{*}$ & 7.095 & 0.312 \\
\hline $\begin{array}{l}\text { Identificación de } \\
\text { valores con fami- } \\
\text { liares en la comuni- } \\
\text { cación por Internet }\end{array}$ & 0.746 & 0.945 & 15.904 & $0.003^{*}$ & 8.343 & 0.214 \\
\hline
\end{tabular}

* $\mathrm{p}<0.05$ diferencia significativa

Fuente: Elaboración propia (2019)

Otro aspecto importante de resaltar al realizar el análisis de estas dos dimensiones es la utilización de Internet por parte de los estudiantes en relación con la dimensión de finalidad de uso de Internet para comunicarse, observándose que ha tenido una alta incidencia de correlaciones con un total de 4 . Siendo su primera relación significativamente con el Internet promueve valores $(p=0.012)$. La segunda correlación positiva y muy alta con la dimensión identificación de valores con compañeros en la comunicación por Internet $(p=0.000)$. La tercera correlación significativa con la dimensión identificación de valores con amigos en la comunicación por Internet $(p=0.028)$ y la cuarta correlación bastante significativa es la dimensión identificación de valores con familiares en la comunicación por Internet $(p=0.003)$.

\section{Discusión y Conclusiones}

Las conclusiones derivadas del estudio planteado y los análisis realizados permiten señalar que se han cumplido la totalidad de las hipótesis propuestas. La presencia de valores sociales se da con fines comunicativos en los estudiantes universitarios, $(p=0.011)$ contrastando así la hipótesis general, lo cual coincide con los aportes de Parra (2010, p. 206), quien defiende que los jóvenes encuentran en Internet un espacio "que los conducen a la producción de ideas, al sostenimiento de diálogos de todo tipo, al encuentro de mensajes que coinciden con sus intereses", es decir a comunicarse, a poner en práctica sus propios valores personales y sociales porque este proceso comunicativo se traduce en beneficios satisfactorios para ellos mismos.

La importancia que los jóvenes le dan a Internet al formar parte de su vida diaria y de lo cotidiano en todos los ámbitos donde se desenvuelven ha caracterizado a que este tenga una alta relevancia, según Bonilla-del-Río, Diego-Mantecón y Lena-Acebo, (2018) en su investigación describen al factor usuario responsable, que incide en valores sociales, como el tener un comportamiento adecuado y respetuoso en las redes, el utilizar los mismos valores de respeto de la vida real en Internet, aspectos que han sido contrastados en nuestra hipótesis (H1) al referir que la presencia de valores sociales se relaciona significativamente con la dimensión relevancia del uso de Internet $(p=0.037<0.05)$.

La utilización de Internet por parte de los usuarios ha ido decreciendo en cuanto a la edad, ya que ahora la utilizan desde el maternal, lo cual está conllevando a que se incremente el uso en años, como lo mencionan Gamito, Aristizabal, Olasolo y Vizcarra, (2017) que la edad de inicio para el uso de Internet es entre 8 y 10 años, lo cual también se ha verificado en la hipótesis $(\mathrm{H} 2)$ de esta investigación que la presencia de valores sociales se relaciona significativamente con los años de uso de Internet en los estudiantes $(p=0.001<0.05)$.

El que se tenga a disposición Internet todo el tiempo, ha conllevado a que muchos de los usuarios utilicen este recurso en cualquier momento del día o noche indistintamente en el lugar que se encuentren al disponer de libre acceso, en su investigación uso de Internet y redes sociales en estudiantes universitarios Molero et al., (2014) refieren que en promedio se conectan tres horas diarias los estudiantes fundamentalmente a la redes sociales, mientras que Fernández, Casal, Fernández-Morante y Cebreiro, (2019) mencionan que la totalidad del alumnado en su investigación se conecta a Internet diariamente, resultados que en este estudio se ha contrastado en la hipótesis (H3), que el uso de Internet en horas a la semana se relaciona con que Internet transmite valores $(p=0.048<0.05)$.

La incursión de las tecnologías de información y comunicación y con ella la llegada de Internet ha dado un giro de más de 360 grados a la educación en general, ya que ha permitido romper fronteras de todo tipo, así como repensar el modo de aprender y enseñar por la ingente cantidad de información que se tiene al alcance en milésimas de segundos, aunado a ello la transmisión de valores sociales que se encuentran implícitos dentro de 
la interacción que se pueda dar en la búsqueda de información o al interactuar de manera sincrónica o asincrónica en la red, para Fernández, Casal, Fernández-Morante y Cebreiro, (2019) mencionan que los estudiantes universitarios de Galicia se conectan a Internet en un 89,4\% para buscar información relaciona con sus estudios, Alvites-Huamaní, (2019) que las tecnológicas son un medio de comunicación, de formación y de interacciones amicales y en la que comparten normas, valores y sentido de pertenencia, lo que se corrobora en la hipótesis (H4) de este estudio que la dimensión finalidad de uso de Internet para obtener información, comunicarse y entretenimiento de los estudiantes se relaciona con que Internet transmite valores sociales $(p=0.03)$.

Este estudio invita a reflexionar sobre cómo Internet se ha convertido en un espacio en el que se transmiten valores sociales, y que precisa de investigaciones y acciones pedagógicas y educativas para abordarlo desde las aulas universitarias. Los estudiantes universitarios hacen un uso indiscutible de esta Red y la formación universitaria debe pensar no solo en términos metodológicos o didácticos, sino también axiológicos, porque el eje vertebral de las sociedades democráticas, justas y responsables se sustenta en valores socialmente compartidos, defendibles y equitativos, por lo que debe impregnar las prácticas docentes de estas cualidades. En este sentido, la práctica docente además de atender a nuevas metodologías y formas de enseñar más atractivas debe centrarse en la transmisión de contenidos de forma axiológicamente adecuada, una necesidad que se hace imprescindible en un momento en el cual la información y los contenidos audiovisuales llegan al usuario sin depurar. Por tanto, y para paliar tal carencia se propone el desarrollo de la ya mencionada competencia crítica que permite al usuario, en este caso al estudiante universitario de diferentes contextos, desarrollar la habilidad que le capacita para diferenciar entre valores, contravalores e información falsa o no verídica, aspectos que ya se están trabajando a través de diversos contextos como los mostrados en el marco teórico en Colombia (Ricardo e Iriarte, 2017) y destaca el Informe publicado por Adams-Becker, Cummins, Davis, et al (2017) y posteriormente, el de 2019 por Alexander, Ashford-Rowe, Barajas-Murphy, et al (2019), en el que se apuesta por el desarrollo e implementación de nuevas metodologías colaborativas y formaciones e-learning.

Además, cabe reseñar que una de las principales limitaciones del estudio se centra en la complejidad por obtener una mayor muestra de los participantes, un elemento que se debe intentar mejorar en futuras investigaciones. Asimismo, indagar si existe alguna diferencia de presencia de valores sociales y el uso de Internet según el género, edad y aspectos sociodemográficos.

\section{Referencias bibliográficas}

Acuña-Caicedo, R.W., Caicedo-Plúa, C.R., Rodríguez-González, A.C., y Figueroa-Morán, G.L. (2017). Importancia de los entornos moocs para la divulgación de conocimientos académicos en entornos universitarios. 3C Tecnología: glosas de innovación aplicadas a la pyme, 6(3), 33-47. doi: http://dx.doi. org/10.17993/3ctecno.2017.v6n3e23.33-47

Adams-Becker, S., Cummins, M., Davis, A., Freeman, A., Hall Giesinger, C., \& Ananthanarayanan, V. (2017). NMC Horizon Report: 2017 Higher Education Edition. Austin, Texas: The New Media Consortium.

Alexander, B., Ashford-Rowe, K., Barajas-Murphy, N., Dobbin, G., Knott, J., McCormack, M.,... Weber, N. (2019), EDUCAUSE Horizon Report: 2019 Higher Education Edition. Washington, DC: Educause.
Álvarez-Arregui, E. (2019). Evolución de la Universidad en la Sociedad del Aprendizaje y la Enseñanza. El valor de las competencias en el desarrollo profesional y personal. Aula Abierta, 48(4), 349-372. doi: https://doi.org/10.17811/rifie.48.4.2019.349-372

Alvites-Huamaní, C.A. (2019). Adolescencia ciberbullying y depresión, riesgos en un mundo globalizado. Revista Científica Electrónica de Educación y Comunicación en la Sociedad del Conocimiento, 19 (1), 210-234.

Anaya, J. P. (2019). Ética de la comunicación en Internet móvil. Madrid: Taurus.

Asher, Y., Stark, A., \& Fireman, G. (2017). Comparing electronic and traditional bullying in embarrassment and exclusion scenarios. Computers in Human Behavior, 76, 26-34. https://doi. org/10.1016/j.chb.2017.06.037

Atrevia (2016). Generación Z. Deusto: Universidad de Deusto. Recuperado de https://goo.gl/NNiwXE

Bauman, Z. (2014). Vivimos en dos mundos paralelos y diferentes: el online y el offline. Clarin.com Recuperado de http://goo.gl/ OH8If5

Beaumont, R. (2009). Research methods y experimental design: A set of notes suitable for seminar use. Introduction to Health Informatics Research Methods. Recuperado de https:/ / bit.ly/37kDskE

Berlanga, I., Gozálvez, V., Renés, P., y Aguaded, I. (2018). Diez años de smartphones. Un análisis semiótico-comunicacional del impacto social de la telefonía móvil. Aula Abierta, 47(3), 299306. doi: https://doi.org/10.17811/rifie.47.3.2018.299-306

Bonilla-del-Río, M., Diego-Mantecón, J. M., y Lena-Acebo, F.J. (2018). Estudiantes Universitarios: prosumidores de recursos digitales y mediáticos en la era de internet. Aula Abierta, 47 (3), 319-326. https: / / doi.org/10.17811/rifie.47.3.2018.319-326

Boyer, E. (2003). Una propuesta para la educación superior del futuro. México: Fondo de Cultura Económico.

Caldeiro-Pedreira, MC. (2014). Alfabetización comunicativa para el desarrollo de la autonomía mediática. Estudio de la competencia mediática de los adolescentes de Lugo (Galicia). Recuperado de http://goo.gl/1UJ4Nm

Caldeiro, M. C. y Aguaded-Gómez, J. (2015). Estoy aprendiendo, no me molestes la competencia mediática como forma de expresión crítica de nativos e inmigrantes digitales. Redes.com, 12. Recuperado de http://goo.gl/2ORkf1

Colina, (2012). La universidad en el contexto de los valores que la identifican. Opción, 28, 69, 546-560.

Colom, T., y Melich, J. C. (1993). Postmodernidad y educación. La teoría de Toffler y la práctica de la C. M. U. Teoría de la Educación. Revista Interuniversitaria, 5, 97-110.

DiMaggio, P., Hartittai, E., Celeste, C., \& Sheffer, S. (2004). Digital inequality; from unequal access to differentiated use. En Neckerman, K. (coord.). Social Inequality. USA, NY: Russell Sage Foundation, 355-400.

Echeverría, J. (2000). Un mundo virtual. Barcelona: Plaza \& Janés Editores S.A.

Fernández, J., Casal, L., Fernández-Morante, C., y Cebreiro, B., (2020). Actitudes y uso de internet y redes sociales en estudiantes universitarios/as de Galicia: implicaciones personales y sociales. Revista Prisma Social, 28 (1), 145-160.

Fombona, J., y Roza Martin, P. (2016). Uso de los dispositivos móviles en educación infantil. Edmetic, 5(2), 158-181.

Garrido-Cabezas, N. (2011). Internet como un nuevo medio social para la comunicación y la participación ciudadana. Revista de comunicación Vivat Academia, 117E, 1375- 1390. https:/ / doi.org/10.15178/va.2011.117E.1375-1390

Gozálvez, V. (2013). La ciudadanía mediática. Una mirada educativa. Madrid: Dykinson. 
Guerra, C., Montiel, I., Molina, N., Escalona, Y., Riquelme, J., y Rojas, L. (2019). Análisis de una escala breve de conductas de riesgo en internet en jóvenes chilenos. Anuario de psicología, $49,32-39$.

Gubern, R. (2000). El eros electrónico. Barcelona: Taurus.

Hassani, S. (2006). Locating digital divides at home, work, and everywhere else. Poetics, 34, 250-272.

Hernández Díaz, J. M., y Eyeang, E. (eds.) (2017). Los valores en la educación de África. De ayer a hoy. Salamanca: Ediciones Universidad de Salamanca.

Ibarra Rius, N., Ballester Roca, J., y Marín, F. (2018). Encrucijadas de la competencia mediática y la ciudadanía: Uso y consumo de aplicaciones educativas. Revista Prisma Social, (20), 92-113. Recuperado de https://bit.ly/353gMUB

Lévy, P. (1999). ¿Qué es la virtualidad? Barcelona: Paidós.

Martínez, M., Esteban, F., y Buxarrais, M.R. (2011). Escuela, profesorado y valores, Revista de Educación, N. ${ }^{\circ}$ Extra. 1, 95-113.

Martínez-Otero Pérez, V. (2019). Claves axiológicas y retos educativos en Iberoamérica. Revista Iberoamericana De Educación, 80(2), 105-127. Recuperado de https:/ / bit.ly/369kPA3

Molero, M., Martos, A., Fernández, F., Barragán, A., y Pérez-Fuentes, M. (2014). Uso de internet y redes sociales en estudiantes universitarios, European Journal of Child Development, 2 (3) 8196. https://doi.org/0.1989/ejpad.v2i3.24

Parra, E. (2010). Las redes sociales de Internet: también dentro de los hábitos de los estudiantes universitarios. Tendencias, XI, 2, 193-207.
Parodi, G., Moreno-de-León, T., Julio, C., \& Burdiles, G. (2019). Google or Gutenberg Generation: Chilean university students' reading habits and reading purposes. Comunicar, 58, 85-94. https:/ / doi.org/10.3916/C58-2019-08

Ricardo-Barreto, C., y Iriarte-Diazgranados, F. (2017). Las TIC en la educación superior. Experiencias de innovación. Colombia: Universidad del Norte editorial. Recuperado de https://bit. ly/37mPQQW

Silva Quiroz, J., y Maturana Castillo, D. (2017). Una propuesta de modelo para introducir metodologías activas en educación superior. Innovación educativa (México, DF), 17(73), 117-131. Recuperado de https:/ / bit.ly/37iWkQV

Smith, M., \& Kollock, P. (Ed.) (2003). Comunidades en el ciberespacio. Barcelona: UOC. Colección Nuevas Tecnologías y Sociedad.

UNESCO. (2015). Replantear la educación. ¿Hacia un bien común mundial? Recuperado de https:/ / bit.ly/39pP5Zu

Web Fundación Universidad de La Rioja. https:/ / bit.ly/2tUKjTl

Wilson, E. (2000). Closing the Digital Divide: An initial review. USA, Washington: internet Policy Institute. 